\title{
IL22 drives cutaneous melanoma cell proliferation, migration and invasion through activation of miR-181/STAT3/AKT axis
}

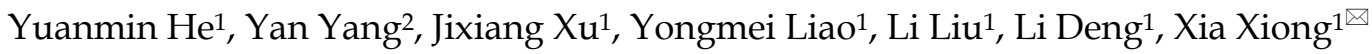 \\ 1. Department of Dermatology, the Affiliated Hospital of Southwest Medical University, Luzhou, Sichuan 646000, China \\ 2. Department of Public Health, Southwest Medical University, Luzhou, Sichuan 646000, China \\ $\square$ Corresponding author: Yuanmin He, Department of Dermatology, the Affiliated Hospital of Southwest Medical University, No 25 of Taiping Road, Luzhou, \\ Sichuan 646000, China. Email: heym88@163.com \\ (1) The author(s). This is an open access article distributed under the terms of the Creative Commons Attribution License (https://creativecommons.org/licenses/by/4.0/). \\ See http://ivyspring.com/terms for full terms and conditions.
}

Received: 2019.10.03; Accepted: 2020.01.31; Published: 2020.02.19

\begin{abstract}
Cutaneous melanoma (CM) is neoplastic growth of melanocytes with strong potential to proliferate and invade, prone to a fatal disease soon which is beyond surgical clearance. The use of regulator involving in antitumor immune responses has been identified as a potential therapeutic option for CM, but still need fully understood at present. Recently, interleukin 22 (IL22), an immune molecule secreted mostly by $\mathrm{CD}^{+} \mathrm{T}$ cells, was reported having functions in a variety of human diseases including encouragement of lung cancer progression, yet, its role in CM is lacking. Here, we first found elevated expression of IL22 in both serum of CM patients and tissues. Up-regulated IL22 significantly promoted cell proliferation, migration and invasion in CM cells deriving from different original culture history. Moreover, in vivo CM model, IL22 treatment caused a significant increase in tumor size. Additionally, we found these effects accompanied by obvious increased miR-181 expression in CM. Importantly, both in vivo and in vitro results revealed that miR-181 downregulation reversed the effects of IL22 on CM cell proliferation, migration, invasion, and CM tumor size as well. Finally, in CM cells deriving from different culture history, we identified STAT3 to be a target gene of miR-181. Higher expression level of IL22 suppressed STAT3 expression, while enhanced expression of $\mathrm{p}-\mathrm{AKT}, \mathrm{p}-\beta$-catenin and MMP4; however, down-regulation of miR-181 reversed these situations. Thus, we conclude that IL22 promotes CM progression by driving miR-181/STAT3/AKT axis.
\end{abstract}

Key words: cutaneous melanoma, IL22, miR-181, STAT3, AKT

\section{Introduction}

Cutaneous melanoma $(\mathrm{CM})$ is one of the most common malignancies with high aggressive potential $^{1}$, and it is estimated that there will be 96,480 new cases of CM and approximately 7,230 deaths from this disease in 2019 in the United States (https://seer. cancer.gov/statfacts $/ \mathrm{html} /$ melan.html). In the last decade, despite evolving progress have been made in identifying new therapeutic options to target cell proliferation and invasion pathways ${ }^{2}$, the potential use of regulator involving in antitumor immune responses and the molecular basis underlying their tumorsuppressed activity are extensively being investigated for providing effective therapeutic approaches for advanced $\mathrm{CM}^{3}, 4$. Since the 1990s, interferon alpha and interleukin (IL)2 have been tested widespread in clinical trials, and increasing numbers of immunotherapeutic approaches have being identified as potential effective options in advanced $\mathrm{CM}$ treatment ${ }^{5}$. However, currently, there is no proven treatment with both high response and low resistance ${ }^{6}$. Thus, the exact immune modulator implicated in the antitumor immune responses on CM aggressiveness need more in-depth studies.

IL22, a immunoregulatory cytokine, is selectively produced by $\mathrm{CD} 4^{+} \mathrm{T}$ cell subsets such as Th17 cells and Th22 cells, innate lymphoid cells, 
natural killer $\mathrm{T}$ cells, $\gamma \delta \mathrm{T}$ cells, and rarely $\mathrm{CD}^{+} \mathrm{T}$ cells ${ }^{7,8}$. IL22 is a distinctive member of IL10 family of cytokines, and seems to act exclusively on epithelial cells with expressing a transmembrane receptor of IL22R1 and IL10R29, 10. The signaling pathway downstream of IL22 has not yet been fully elucidated. STAT3 cascade, inducing phosphorylation in several kinases, was considered to be a mediator of IL22-induced effects in some studies ${ }^{11,12}$. Individual miRNAs involving in modulating $\mathrm{CD}^{+} \mathrm{T}$ cell responses also have been found critical for activation of IL22 and STAT3 signaling13,14.

Previous studies have shown that IL-22 plays critical roles through its receptor in promotion of antimicrobial immunity, tissue repair and inflammation ${ }^{15}$. Recently, IL-22 has been closely related to the initiation and progression of cancer ${ }^{16}$. Remarkably, IL-22 signaling was identified as a critical regulator in cell proliferation, migration and apoptosis, that could be hijacked by aggressive cancers to enhance tumor growth and metastasis ${ }^{12}, 17$. However, the potential role of IL-22 in CM and underlying mechanism involved in it remain unclear.

In the present study, we noticed that IL-22 was up-regulated in both serum of CM patients and CM tissues, accompanying by significantly increased miR-181 expression. Moreover, we confirmed that up-regulated IL22 significantly promoted cell proliferation, migration and invasion in $\mathrm{CM}$ cells deriving from different original culture history, as well as caused a significant increase in tumor size in vivo CM model; however, miR-181 downregulation reversed the effects of IL22 in both vivo and vitro models. Finally, we identified STAT3 to be a target gene of miR-181, which involving in enhanced expression of p-AKT, p- $\beta$-catenin and MMP4.

\section{Methods and Materials}

\section{Patients and samples}

$\mathrm{CM}$ tissues, adjacent normal tissues and peripheral blood were collected from 30 patients diagnosed with $\mathrm{CM}$ according to clinical and histological manifestation in department of dermatology, the Affiliated Hospital of Southwest Medical University (Based on the expression of interleukin 22 in normal human tissues and other tumor tissues obtained from published report 18,19 , we statistical estimated sample size in our study, and found that 30 cases enrolled in both patient group and health group could meet the statistical requirement.). They are 40-60 years old with 17 male and 13 female. 13 of them were diagnosed as stage I-II CM and 17 of them were diagnosed as stage III-IV CM. Control serum were obtained from corresponding 30 healthy controls with matched age and sex from the Affiliated Hospital of Southwest Medical University. Each sample was snap-frozen using liquid nitrogen, followed by storing at a temperature of $-80^{\circ} \mathrm{C}$ until processed. The investigational protocol was approved by the Ethics Committee of the Affiliated Hospital of Southwest Medical University according to the Declaration of Helsinki Principles.

\section{Cell culture and transfection}

CM cell lines (A375 and M14) were obtained from Chinese Academy of Science and cultured in RPMI1640 (Gibco, Grand Island, NY, USA) medium containing 10\% FBS (Gibco, Grand Island, NY, USA) with $100 \mathrm{U} / \mathrm{mL}$ penicillin-streptomycin (Invitrogen, Carlsbad, CA, USA). Cells were cultured at $37^{\circ} \mathrm{C}$ in $5 \%$ CO2. IL22 were obtained from Sigma Chemical Co (St. Louis, MO, USA). Sh-miR-181 and control oligonucleotide were obtained from Genepharma (Shanghai, China). The oligonucleotides were transfected into A375 and M14 cells (200 nmol per well) using Lipo3000 (Invitrogen, Carlsbad, CA, USA).

\section{3-(4,5-dimethylthiazol-2-yl)-2, 5-diphenyltetrazolium bromide (MTT) assay}

A total of 3000 cells/well were seeded into 96-well plates and cultured for $12 \mathrm{~h}$ followed by transfection with sh-miR-181 or control oligonucleotide. After $24 \mathrm{~h}$ of transfection, IL22 (Sigma, St. Louis, MO, USA) was added into each well and cultured for $24 \mathrm{~h}$, MTT assay was used to assess the cell viability. $0.5 \%$ MTT (Sigma, USA) was added to the culture medium at $37^{\circ} \mathrm{C}$ for $4 \mathrm{~h}$. The supernatant was removed and DMSO (Gibco, CA, USA) was added into each well. After that, the absorption at $490 \mathrm{~nm}$ was evaluated using microplate reader (BioRad, CA, USA).

\section{ELISA}

Human IL22 Quantikine ELISA kits was used to analyze patient's serum samples, following the manufacturer's instructions (R\&D Systems).

\section{Clonogenic assay}

The cells treated with indicated conditions and then seeded in 12-well plates (100/well). After incubated for 2 weeks, crystal violet $(0.05 \%$, Beyotime, Shanghai, China) was used to stain the colonies. Colonies with sizes $\geq 1 \mathrm{~mm}$ were counted.

\section{Wound healing}

The cells treated with indicated conditions and then seeded in 6-well plates $\left(1 \times 10^{4} /\right.$ well $)$. After cultured in serum-free medium for 24 hours, the cell monolayer was wounded with a $10 \mu$ pipette tips. Fresh medium was replaced. The wound-closing 
procedure was observed for 48 hours and photographs were taken at $0 \mathrm{~h}, 48 \mathrm{~h}$ after wounding to determine the wound-closing procedure.

\section{Transwell assay}

Cell invasion ability was detected by the transwell assay (Millipore, MA, USA). Cells were treated with indicated conditions and then seeded on the upper insert coated with 2\% Matrigel (BD Biosciences, NY, USA) in 24-well plates (5000/well). The upper insert was filled with medium lacking serum and the lower chamber was filled with $600 \mu \mathrm{L}$ DMEM supplemented with $10 \%$ FBS. After $24 \mathrm{~h}$ of incubation, cells invaded to the lower chambers were fixed with methanol, stained with crystal violet.

\section{Quantitative real-time PCR}

Total RNAs were extracted using Trizol Reagent (Invitrogen, CA, USA) according to the manufacturer's instruction. $1 \mu \mathrm{g}$ total RNAs was reversely transcribed to cDNA using a RNA PCR Kit (Takara, Dalian, China) which was used as a PCR template. To detect gene expression, quantitative real-time PCR (qRT-PCR) was performed using an iCycler iQ System with the IQ SYBR Green Super Mix (BioRad, CA, USA) according to manufacturer's instructions. Small endogenous nuclear U6 snRNA was used as internal control for normalization of miRNA and GAPDH for mRNAs. The relative gene expression levels were calculated using $(2-\Delta \Delta \mathrm{Ct})$ method.

\section{Western blot}

The protein samples were extracted from the A375 and M14 cells using a lysis buffer. Amounts of $40 \mu \mathrm{g}$ protein was separated in $10 \%$ sodium dodecyl sulfate-polyacrylamide gel electrophoresis and transferred to polyvinylidene fluoride membranes. The blots were incubated with 5\% skimmed milk in PBS for $2 \mathrm{~h}$ to block non-specific binding followed by probing with primary antibodies (Cell Signaling Technology, USA) overnight at $4^{\circ} \mathrm{C}$. After washed with TBST, the blots were incubated at room temperature for $90 \mathrm{~min}$ with horseradish peroxidaseconjugated goat anti-rabbit antibodies (Cell Signaling Technology, USA). GAPDH (Cell Signaling Technology, USA) was used as an internal control. Finally, the blot was treated with ECL plus reagent (Pierce, Rockford, IL, USA) and detected using a chemiluminescence detection kit (KPL, Gaithersburg, MD).

\section{Luciferase assay}

The wild-type and mutated 3'UTRs of STAT3 were subcloned into the pGL3 vector (Promega, WI, USA). Cells were co-transfected with the plasmid constructs of pGL3-STAT3-3'UTR or pGL3-STAT3-
3'UTR-mut and ad-miR-181 or ad-control using Lipofectamine 3000 (Invitrogen, CA, USA). Subsequently, cells were transfected with $0.1 \mu \mathrm{g}$ PRL-TK (TK-driven Renilla luciferase expression vector) as an internal control. Luciferase activities were measured $48 \mathrm{~h}$ after transfection with a dual luciferase reporter assay kit (Promega, WI, USA).

\section{In vivo study}

$30 \mathrm{Balb} / \mathrm{c}$ nude male mice were maintained under a specific pathogen-free condition and randomly divided into three groups. All experiments were approved and carried out according to the guidelines of the Ethics Committee of The Affiliated Hospital of Southwest Medical University. A375 and M14 cells in the amount of $1 \times 10^{6}$ were subcutaneously injected into the flank region of the mice. IL22 dissolved in normal saline was administered to these mice everyday at the dose of $50 \mathrm{mg} / \mathrm{kg}$ for 10 days before tumor inoculation until the end of the study. The mice in IL22+sh-miR-181 group were tail injected with adi-sh-miR-181 after IL22 administration. The tumor sizes were measured every two days using a caliper. The mice were sacrificed at day 30 and tumors were excised. Mice were placed in a Plexiglas chamber with 5\% isoflurane (VetOne, Shanghai, China) for $5 \mathrm{~min}$, and decapitated when fully sedated, as measured by a lack of active paw reflex. A part of the issues was placed in $10 \%$ formalin for histological and the remaining was frozen in $-80^{\circ} \mathrm{C}$.

\section{Statistical analysis}

All statistical analyses were performed with GraphPad Prism (GraphPad software 3.0; San Diego, CA, USA). All the data are presented as the means \pm SD. One-way ANOVA was used to assess the difference between multiple groups. Differences between two groups were analyzed by the Student's t-test. $\mathrm{P}<0.05$ was considered as statistical significance.

\section{Results}

\section{Elevated IL22 is detected in both serum of CM patient and CM tissue}

We first detected expression levels of IL22 in tissues from CM and adjacent normal skin, as well as in serum from $\mathrm{CM}$ patients and health control. As shown in Figure 1A and 1B, expression levels of IL22 dramatically elevated in both serum and tissues from CM patients. Consistently, IHC analysis also showed obviously enhanced expression of IL22 in CM tissues (Figure 1C). These results indicate that IL22 is up-regulated in CM. 

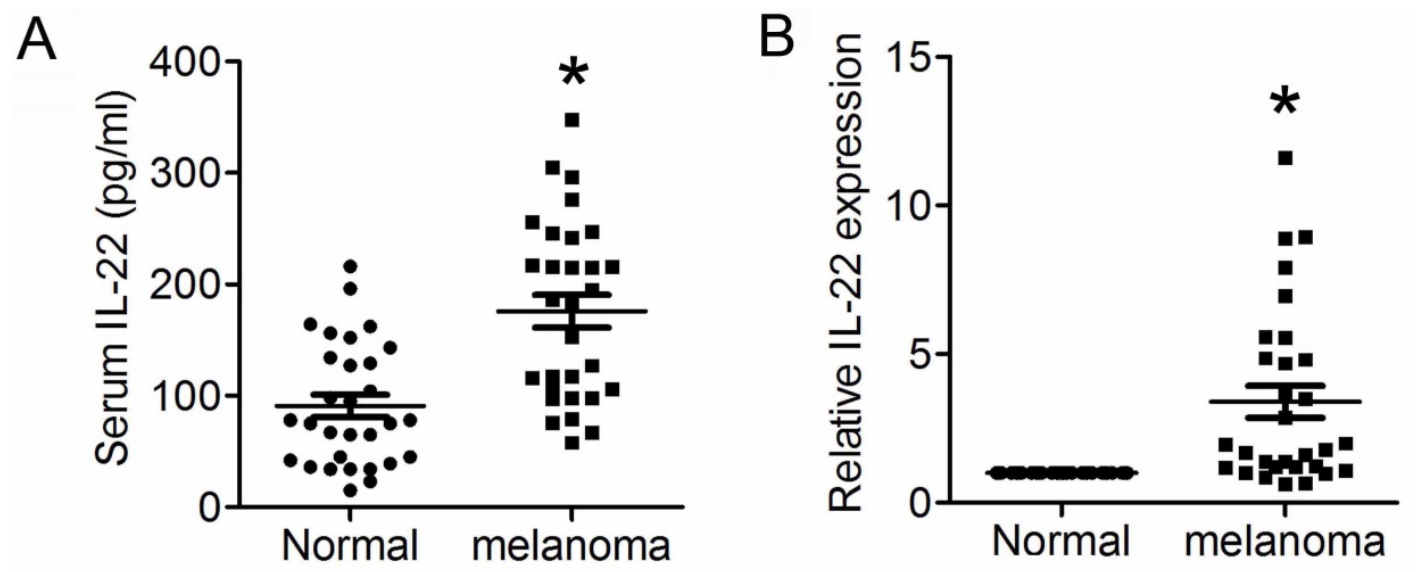

C

\section{Normal}

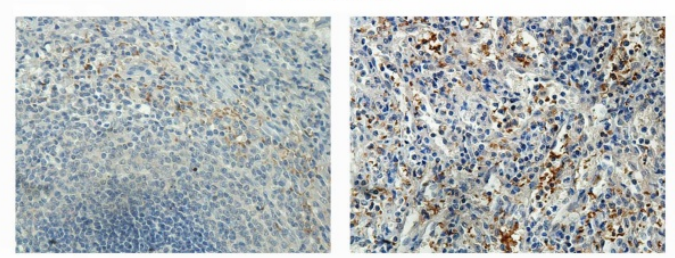

melanoma

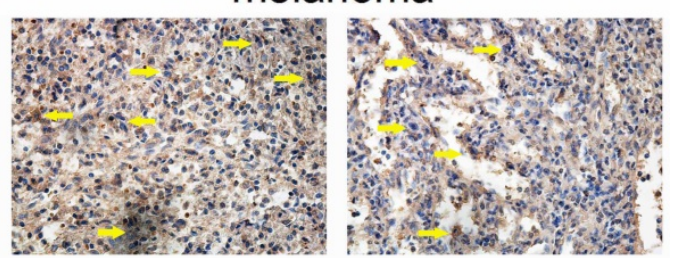

Figure 1. Elevated IL22 in serum of CM patient and CM tissue. (A) IL22 expression in serum of CM patients evaluated by ELISA. (B) IL22 mRNA in CM tissues detected by qRT-PCR. (C) IL22 protein expression in CM tissues detected by IHC $(\times 200)$. $* P<0.05$ verse normal group.
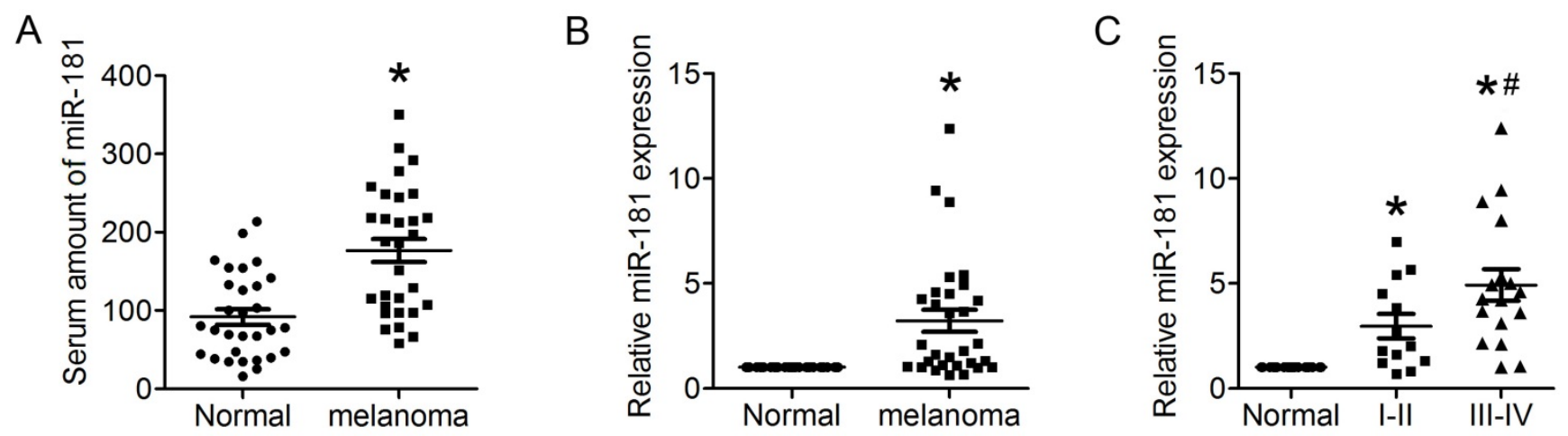

Figure 2. Up-regulated miR-181 in serum of CM patient and CM tissue. (A) Expression of miR-181 in serum of CM patients detected by qRT-PCR. (B) Expression of miR-181 in CM tissues detected by qRT-PCR. (C) Expression of miR-181 in stage I-II CM tissues and stage III-IV CM tissues. *P<0.05 verse normal group, $\# P<0.05$ verse stage I-II CM group.

miR-181 is up-regulated in the serum of melanoma patient and the melanoma tissue

IL22 have been identified as an effecter secreted by $\mathrm{CD}^{+} \mathrm{T}$ cells that promotes lung tumorigenesis ${ }^{20}$. Recent studies found miR-181 with a clear effect on $\mathrm{CD}^{+} \mathrm{T}$ cell development and homeostasis, and involved in cellular apoptosis, differentiation and tumorigenesis ${ }^{21}, 22$. To identify whether miR-181 expression is associated with up-regulated expression of IL22 in CM, qRT-PCR was preformed to evaluate the relative mRNA expression of miR-181 in both serum and tissues from CM patients. The present results demonstrated significantly increased levels of miR-181 in both serum (Figure 2A) and tissues (Figure 2B) from CM patients. Moreover, expression levels of
miR-181 in tissues from stage III-IV CM were remarkably higher than that from stage I-II CM (Figure 2C).

\section{IL22 promotes cell proliferation, migration, invasion, and and clonogenic ability in CM cells via up-regulated miR-181}

To identify the roles of IL22 and miR-181 in CM progression, we first preformed sh-miR-181 oligonucleotide transfection to establish stable low expression of miR-181 in selected CM cell lines (including A375 and M14) deriving from primary $\mathrm{CM}^{23}$ and metastatic $\mathrm{CM}^{24}$. As shown in Figure $3 \mathrm{~A}$, IL22 treatment notably increased expression of miR-181 in A375 and M14, while sh-miR-181 
transfection significantly reduced expression of miR-181. Next, we evaluated the effect of IL22 and miR-181 on cell proliferation, migration, invasion and and clonogenic ability in A375 and M14 cells. The results indicated that IL22 treatment promoted capabilities of cell proliferation (Figure 3B), migration (Figure 3C), invasion (Figure 3D), and clonogenic ability (Figure 3E) in A375 and M14 cells. Reversely, miR-181 downregulation significantly suppressed IL22-induced cell proliferation (Figure 3B), migration (Figure 3C), invasion (Figure 3D), and clonogenic ability (Figure 3E) in A375 and M14 cells. These results identify that IL22 accelerated CM cells progression through up-regulated miR-181.

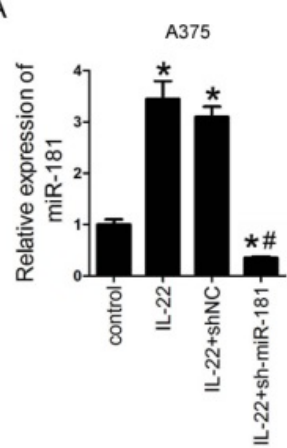

B

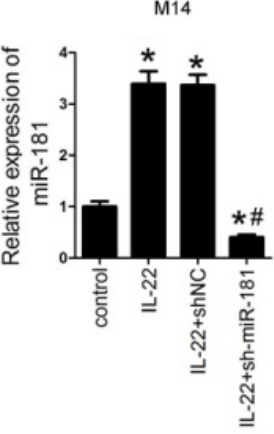

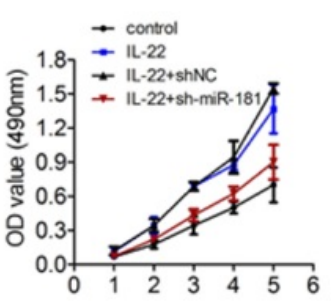
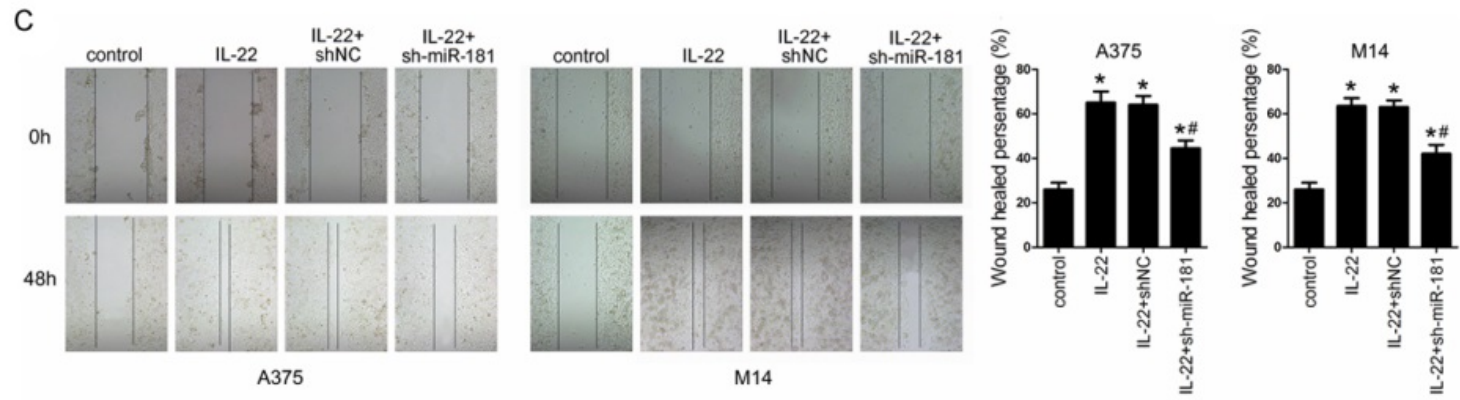

D
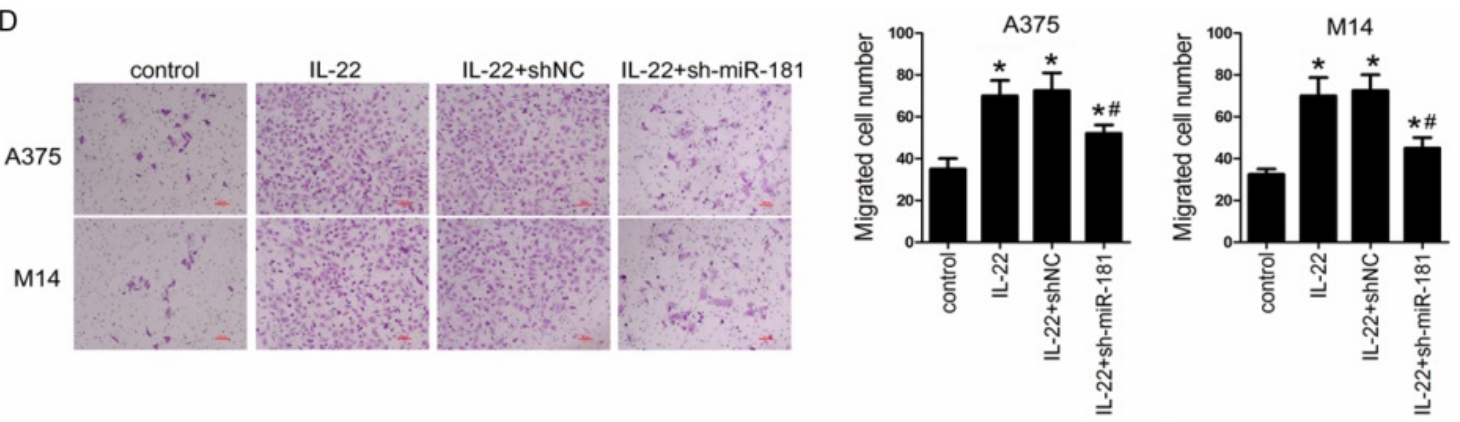

E
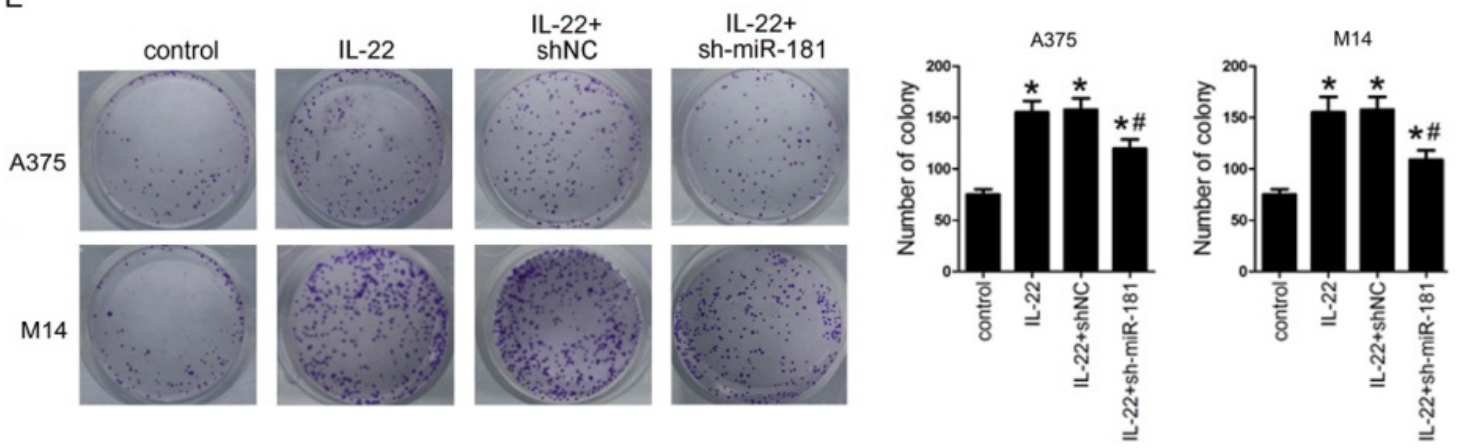

Figure 3. IL22 promotes cell proliferation, migration, invasion, and clonogenic ability in A375 and M14 cells via up-regulated miR-181. (A) Expression of miR-181 in A375 and M14 cells under IL22 and ad-sh-miR-181 treatment. (B) Cell proliferation in A375 and M14 cells detected by MTT. (C) Cell migration in A375 and M14 cells detected by wound healing $(\times 100)$. (D) Cell invasion in A375 and M14 cells detected by transwell assay $(\times 200)$. (E) Clonogenic ability in $A 375$ and M14 cells. $* P<0.05$ verse control group, $\# P<0.05$ verse IL $22+$ shNC group. 


\section{IL22-induced miR-181 upregulation targets STAT3/AKT pathway}

To gain insight into the mechanism underlying the effects of IL22-induced miR-181 upregulation on CM progression, potential gene of miR-181 targeted was predicted by bioinformatic algorithms (Targetscan v7.2), and STAT3 was selected as a target (Figure 4A). To further confirm whether miR-181 directly targets STAT3, a firefly luciferase reporter was constructed containing a wild type or mutated type fragment of the 3'-UTR of STAT3 mRNA. The wild type or mutated type luciferase reporters were co-transfected into A375 and M14 cells with ad-miR-181 or ad-control. The data showed that co-expression of ad-miR-181 with wild type $3^{\prime} \mathrm{UTR}$ but not with mutant 3'UTR significantly inhibited the luciferase activity in A375 and M14 (Figure 4B). In addition, qRT-PCR was carried out to verify the result; as shown in Figure 4C, STAT3 mRNA expression was remarkably inhibited by miR-181 overexpression and recovered by depletion of miR-181. Since STAT3/Akt signaling axis has been identified as crucial step in cancer development and progression, and been found to be a mediator of IL22-induced effects as well12, 25, 26, we detected the expression of downstream proteins in STAT3 signing including $\mathrm{p}$-AKT, $\mathrm{p}-\beta$-catenin, Western blot assay revealed that IL22 treatment significantly inhibited the expression of STAT3, while elevated expression of p-AKT, and matrix metalloproteinase (MMP)-4; however, miR-181 knockdown significantly reversed these effects of IL22 on STAT3/Akt pathway (Figure 4D). These findings indicate that IL22-induced CM progression is regulated, at least partially, by activation of miR-181/STAT3/Akt pathway.
A

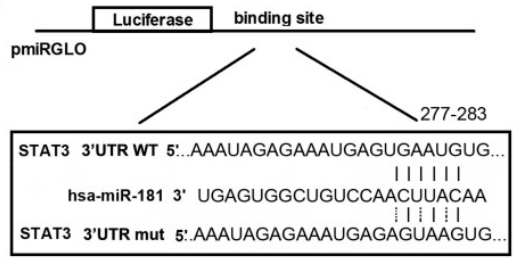

D

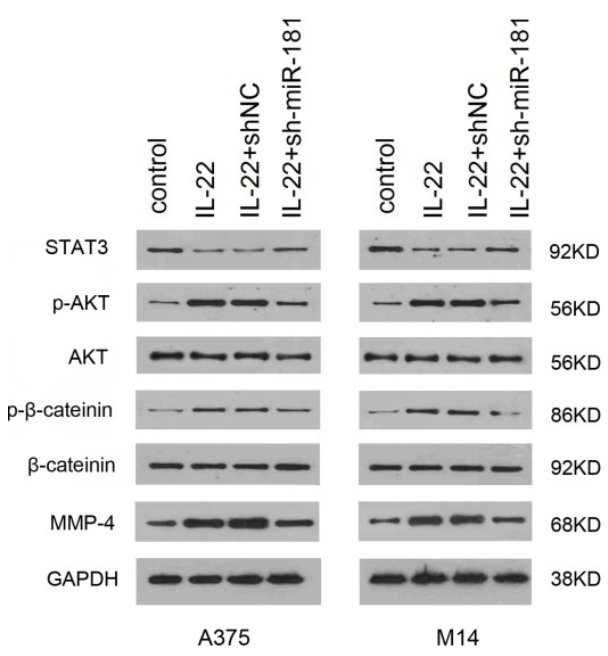

B
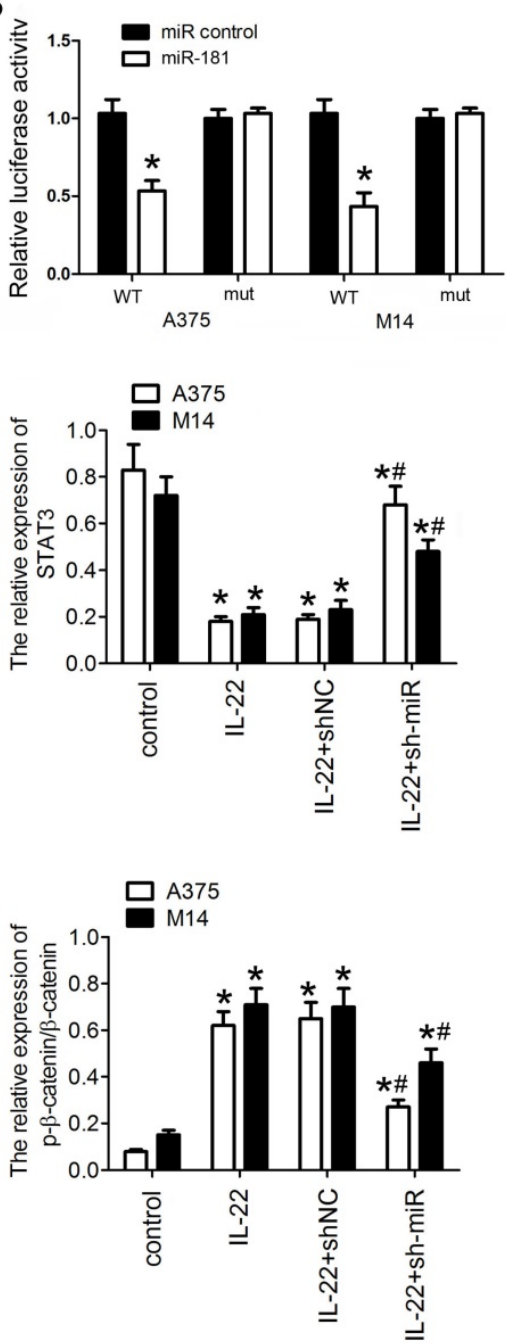

C
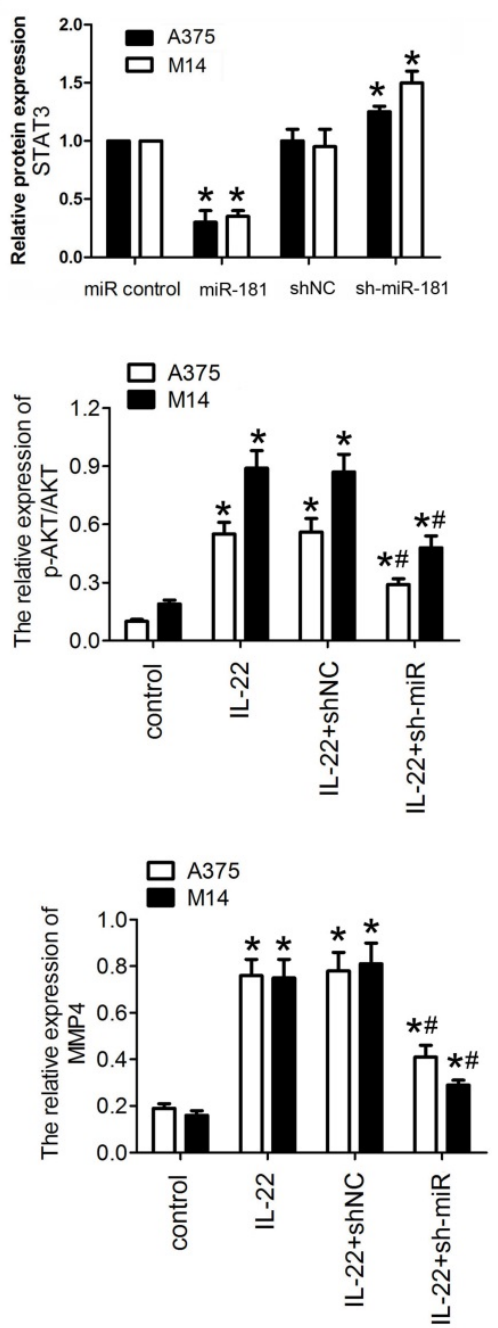

Figure 4. STAT3/AKT branch is activated in A375 and M14 cells under IL22 teatment. (A) The target sequence of miR-181 in 3'UTR region of STAT3. (B) Luciferase activities of reporter vectors in A375 and M14 cells. (C) Expression of STAT3 in A375 and M14 cells under ad-sh-miR-181 treatment. (D) Expression of p-AKT, p- $\beta$-catenin, and MMP-4 in A375 and M14 cells under IL22 and ad-sh-miR-181 treatment. *P<0.05 verse control group, $\#$ P $<0.05$ verse IL22+shNC group. 

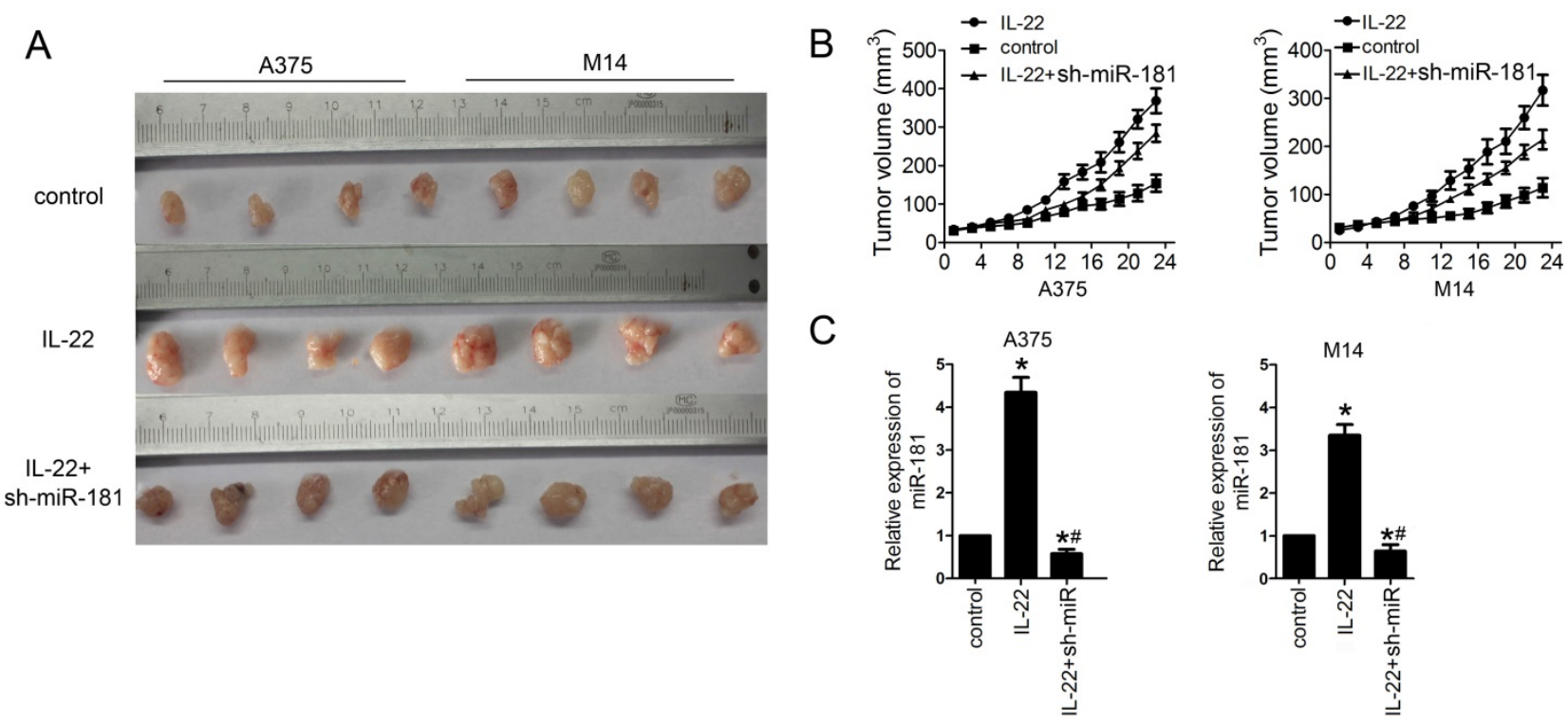

Figure 5. IL22 promotes tumor growth via miR-181 upregulation in CM xenograft models. (A, B) Tumor growth in different CM xenograft models under IL22 and ad-sh-miR-181 treatment. (C) Expression of miR-181 in tumor tissues from different CM xenograft models. ${ }^{*} P<0.05$ verse control group, $\# P<0.05$ verse IL22 group.

\section{IL22 promotes CM growth via miR-181 upregulation in vivo}

Finally, we examined the effects of IL22 on CM progression in vivo. For this reason, models of CM xenograft in nude mice were developed by subcutaneously injection of A375 and M14 cells. As shown in Figure 5A and 5B, IL22 treatment significantly promoted the tumor growth in $\mathrm{CM}$ models; however, sh-miR-181 treatment notably suppressed the effects of IL22 on CM mice. Moreover, qRT-PCR assay results showed that IL22 treatment indeed increased expression of miR-181 in tumor tissues from CM mice (Figure $5 \mathrm{C}$ ).

\section{Discussion}

Previously, IL-22 expression was restricted to innate and adaptive immune cells, while the IL-22R seems to be confirmed in non-hematopoietic cells of the skin, lung, kidney and liver ${ }^{27,28}$. Recently study has revealed high expression levels of IL22 in both lung tumors and serum of patients with lung cancer, promoteing Kras mutant lung tumorigenesis ${ }^{20}$. Cutaneous melanoma is obviously different from ocular melanoma and mucosal melanoma in epidemiological features, clinical characteristics, treatment and outcomes ${ }^{29}, 30$. Although the direct impact and molecular mechanisms of interleukin 22 on human malignancies had been extensively investigated in a great number of previous studies, few report aim at the direct role of interleukin 22 on cutaneous melanoma. To our knowledge, this is the first report show that IL22 promoted cutaneous melanoma cell proliferation, migration, invasion, and increase tumor size in vivo as well.
In the present study, we found that expression of IL22 were significantly higher in CM tissue and serum of CM patient. Although IL-22 was frequently observed in both squamous cell carcinomas and basal cell carcinomas, and was confirmed with obvious impacts on them; to our knowledge, this is first report for detailed associations between IL22 and CM. In the present study, we also evaluated the IL22 mRNA expression level from two online TCGA databases (UALCAN cancer database: http://ualcan.path.uab. edu/index.html; and The Human Protein Atlas, www.proteinatlas.org), however, both of these databases have no data about it. Since IL22 was found mainly produced by $\mathrm{CD}^{+} \mathrm{T}$ cells in tumor-bearing lung tissues, with a key role in modulation of pro-tumor inflammatory responses in lung cancer ${ }^{20}$, our results suggest the probability of IL22 expression being related to dysfunction of tumor-related $\mathrm{CD} 4^{+} \mathrm{T}$ cells in CM. Interestingly, as a previous study shown, naive tumor-specific $\mathrm{CD} 4^{+} \mathrm{T}$ cells could differentiate into cytotoxic $\mathrm{T}$ cells for melanoma clearance ${ }^{31}$, potentially supporting our opinion. Our data also showed that IL22 treatment promoted cell proliferation, migration, invasion, and colony formation in primary $\mathrm{CM}$ cell line $\mathrm{A} 375$, and in metastatic CM cell line M14 as well. Consistently, IL22 treatment significantly promoted the tumor growth in vivo $\mathrm{CM}$ models. These data strongly suggest the suppress effects of IL22 on CM progression.

There are growing evidences that altered expression of miRNAs is strongly linked with carcinogenesis and progression of human malignancies including $\mathrm{CM}^{32}$. Additionally, previous researches have linked distinct sets of miRNAs with 
specific functions of IL-22 in biological processes ${ }^{33,34}$. In the present study, we found up-regulated miR-181 accompanied by significantly increased expression levels of miR-181 in both CM serum and tissues. Moreover, expression levels of miR-181 in stage III-IV $\mathrm{CM}$ tissues were remarkably higher than stage I-II CM. Importantly, we showed down-regulated miR-140-3p significantly suppressed IL22-induced cell proliferation, migration, invasion, and colony formation in A375 and M14 cells. Although miR-181 miRNA family was report with clear effects on $\mathrm{CD} 4{ }^{+} \mathrm{T}$ cell development and homeostasis, at least partly, similar to the functions as IL-22 shown 35 ; our results demonstrate that miR-181 could be employed as a prognostic biomarker for $\mathrm{CM}$ development, as well as a predictor for $\mathrm{CM}$ outcome.

It has been identified that effect of IL22 on tumor progression is mediated through STAT3 signaling, inducing phosphorylation of kinases AKT and Erk1/220,36. However, the role of IL22 and miR-181 in $\mathrm{CM}$ progression is not fully understood. In the current study, we first examined the potential target of miR-181 in CM cell. Both bioinformatics analysis and luciferase reporter assay identified STAT3 as the target gene of miR-181 in CM cells. Moreover, IL22 treatment significantly inhibited the expression of STAT3, while miR-181 knockdown significantly reversed this effect. Furthermore, IL22 treatment elevated expression of $\mathrm{p}$-AKT, $\mathrm{p}$ - $\beta$-catenin and MMP-4; however, miR-181 knockdown significantly inhibited phosphorylation of Akt and $\beta$-catenin, and expression of MMP-4 as well. Although our results showed IL-22 treatment indeed as an activator of STAT3/Akt signing pathway, interestingly, we noticed that the activation of STAT3/Akt pathway in CM cells was mainly mediated directly by miR- 181 . Be similarly to this result, other study also indicated an over-expressed miR-181a in $\mathrm{CD}^{+} \mathrm{T}$ cells could induce phosphorylation of ERK ${ }^{37}$. Previous studies have identified inhibition of $\beta$-catenin signaling and matrix metalloproteinase contributing suppressed roles in both migration and invasion of melanoma cells ${ }^{38}, 39$. Consistently, we observed increased expression of p- $\beta$-catenin and MMP- 4 in $\mathrm{CM}$ cells under IL22 treatment, revealing the underlying mechanism of IL22-induced CM progression. Interestingly, although many studies have linked diverse subset of MMP to invasion of $\mathrm{CM}^{40-42}$, to our knowledge, this is the first report for the role of MMP-4 in CM progression; however, the exact molecular mechanisms implicated in it require additional mechanistic studies.

In summary, our findings provide a new insight into the mechanisms underlying IL22-induced CM progression. Given the potent pro-tumor value of IL22 and miR-181 in CM, them may serve either as prognostic biomarkers or as potential therapeutic strategies for $\mathrm{CM}$ patients.

\section{Acknowledgements}

This work was supported by Scientific Research Foundation for the Doctor in Affiliated Hospital of Southwest Medical University.

\section{Authors' contributions}

Study conception and design: Y.H. and X.X.; acquisition of the data: Y.Y., L.L., J.X. and Y.L.; analysis and interpretation of the data: Y.Y., L.D. and Y.L.; article drafting and revision: Y.H., J.X. and X.X.

\section{Competing Interests}

The authors have declared that no competing interest exists.

\section{References}

1. Schadendorf D, van Akkooi ACJ, Berking C, et al. Melanoma. Lancet.2018; 392: 971-984.

2. Long GV, Fung C, Menzies AM, et al. Increased MAPK reactivation in early resistance to dabrafenib/trametinib combination therapy of BRAF-mutant metastatic melanoma. Nat Commun.2014; 5: 5694.

3. Umansky V, Sevko A. Melanoma-induced immunosuppression and its neutralization. Semin Cancer Biol.2012; 22: 319-326.

4. Garcia-Diaz A, Shin DS, Moreno BH, et al. Interferon Receptor Signaling Pathways Regulating PD-L1 and PD-L2 Expression. Cell Rep.2017; 19: 1189-1201.

5. Snyder A, Zamarin D, Wolchok JD. Immunotherapy of Melanoma. Prog Tumor Res.2015; 42: 22-29.

6. Corrie P, Hategan M, Fife K, et al. Management of melanoma. Br Med Bull.2014; 111: 149-162.

7. Eyerich S, Eyerich K, Pennino D, et al. Th22 cells represent a distinct human T cell subset involved in epidermal immunity and remodeling. J Clin Invest.2009; 119: 3573-3585.

8. Trifari S, Kaplan CD, Tran EH, et al. Identification of a human helper T cell population that has abundant production of interleukin 22 and is distinct from $\mathrm{T}(\mathrm{H})-17, \mathrm{~T}(\mathrm{H}) 1$ and $\mathrm{T}(\mathrm{H}) 2$ cells. Nat Immunol.2009; 10: 864-871.

9. Zenewicz LA, Flavell RA. Recent advances in IL-22 biology. Int Immunol.2011; 23: $159-163$

10. Sonnenberg GF, Fouser LA, Artis D. Border patrol: regulation of immunity, inflammation and tissue homeostasis at barrier surfaces by IL-22. Nat Immunol.2011; 12: 383-390.

11. Lejeune D, Dumoutier L, Constantinescu S, et al. Interleukin-22 (IL-22) activates the JAK/STAT, ERK, JNK, and p38 MAP kinase pathways in a rat hepatoma cell line. Pathways that are shared with and distinct from IL-10. J Biol Chem.2002; 277: 33676-33682.

12. Khare V, Paul G, Movadat O, et al. IL10R2 Overexpression Promotes IL22/STAT3 Signaling in Colorectal Carcinogenesis. Cancer Immunol Res.2015; 3: 1227-1235.

13. Escobar T, Yu CR, Muljo SA, et al. STAT3 activates miR-155 in Th17 cells and acts in concert to promote experimental autoimmune uveitis. Invest Ophthalmol Vis Sci.2013; 54: 4017-4025.

14. Palin AC, Ramachandran V, Acharya S, et al. Human neonatal naive CD4+ T cells have enhanced activation-dependent signaling regulated by the microRNA miR-181a. J Immunol.2013; 190: 2682-2691.

15. Wolk K, Witte E, Witte K, et al. Biology of interleukin-22. Semin Immunopathol.2010; 32: 17-31.

16. Guillon A, Gueugnon F, Mavridis K, et al. Interleukin-22 receptor is overexpressed in nonsmall cell lung cancer and portends a poor prognosis. Eur Respir J.2016; 47: 1277-1280.

17. He W, Wu J, Shi J, et al. IL22RA1/STAT3 Signaling Promotes Stemness and Tumorigenicity in Pancreatic Cancer. Cancer Res.2018; 78 : 3293-3305.

18. Voigt C, May P, Gottschlich A, et al. Cancer cells induce interleukin-22 production from memory CD4(+) T cells via interleukin-1 to promote tumor growth. Proc Natl Acad Sci U S A.2017; 114: 12994-12999.

19. $\mathrm{Li} \mathrm{H}$, Zhang $\mathrm{Q}, \mathrm{Wu} \mathrm{Q}$, et al. Interleukin-22 secreted by cancer-associated fibroblasts regulates the proliferation and metastasis of lung cancer cells via the PI3K-Akt-mTOR signaling pathway. Am J Transl Res.2019; 11: 4077-4088.

20. Khosravi N, Caetano MS, Cumpian AM, et al. IL22 Promotes Kras-Mutant Lung Cancer by Induction of a Protumor Immune Response and Protection of Stemness Properties. Cancer Immunol Res.2018; 6: 788-797. 
21. Henao-Mejia J, Williams A, Goff LA, et al. The microRNA miR-181 is a critical cellular metabolic rheostat essential for NKT cell ontogenesis and lymphocyte development and homeostasis. Immunity.2013; 38: 984-997.

22. Meng F, Henson R, Lang $M$, et al. Involvement of human micro-RNA in growth and response to chemotherapy in human cholangiocarcinoma cell lines. Gastroenterology.2006; 130: 2113-2129.

23. Giard DJ, Aaronson SA, Todaro GJ, et al. In vitro cultivation of human tumors: establishment of cell lines derived from a series of solid tumors. J Natl Cancer Inst.1973; 51: 1417-1423.

24. Chee DO, Boddie AW, Roth JA, et al. Production of melanoma-associated antigen(s) by a defined malignant melanoma cell strain grown in chemically defined medium. Cancer Res.1976; 36: 1503-1509.

25. Rojanasakul Y. Linking JNK-STAT3-Akt signaling axis to EZH2 phosphorylation: a novel pathway of carcinogenesis. Cell Cycle.2013; 12: 202-203.

26. Blando JM, Carbajal S, Abel E, et al. Cooperation between Stat3 and Akt signaling leads to prostate tumor development in transgenic mice. Neoplasia.2011; 13: 254-265.

27. Tachiiri A, Imamura R, Wang Y, et al. Genomic structure and inducible expression of the IL-22 receptor alpha chain in mice. Genes Immun.2003; 4: 153-159.

28. Wolk K, Kunz S, Witte E, et al. IL-22 increases the innate immunity of tissues. Immunity.2004; 21: 241-254.

29. Teh YL, Goh WL, Tan SH, et al. Treatment and outcomes of melanoma in Asia: Results from the National Cancer Centre Singapore. Asia Pac J Clin Oncol.2018; 14: e95-e102.

30. Sula B, Ucmak F, Kaplan MA, et al. Epidemiological and clinical characteristics of malignant melanoma in Southeast Anatolia in Turkey. Pan Afr Med J.2016; 24: 22.

31. Xie Y, Akpinarli A, Maris C, et al. Naive tumor-specific CD4(+) T cells differentiated in vivo eradicate established melanoma. J Exp Med.2010; 207: 651-667.

32. Saldanha G, Potter L, Lee YS, et al. MicroRNA-21 expression and its pathogenetic significance in cutaneous melanoma. Melanoma Res.2016; 26: 21-28.

33. Escobar TM, Kanellopoulou C, Kugler DG, et al. miR-155 activates cytokine gene expression in Th17 cells by regulating the DNA-binding protein Jarid 2 to relieve polycomb-mediated repression. Immunity.2014; 40: 865-879.

34. Mei Z, Zhou L, Zhu Y, et al. Interleukin-22 promotes papillary thyroid cancer cell migration and invasion through microRNA-595/Sox17 axis. Tumour Biol.2016; 37: 11753-11762.

35. Li QJ, Chau J, Ebert PJ, et al. miR-181a is an intrinsic modulator of T cell sensitivity and selection. Cell.2007; 129: 147-161.

36. Madonna S, Scarponi C, Morelli M, et al. SOCS3 inhibits the pathological effects of IL-22 in non-melanoma skin tumor-derived keratinocytes. Oncotarget.2017; 8: 24652-24667.

37. Li G, Yu M, Lee WW, et al. Decline in miR-181a expression with age impairs $\mathrm{T}$ cell receptor sensitivity by increasing DUSP6 activity. Nat Med.2012; 18: 1518-1524.

38. Vaid M, Singh $T$, Prasad $R$, et al. Therapeutic intervention of proanthocyanidins on the migration capacity of melanoma cells is mediated through PGE2 receptors and beta-catenin signaling molecules. Am J Cancer Res.2015; 5: 3325-3338

39. Serini S, Zinzi A, Ottes Vasconcelos R, et al. Role of beta-catenin signaling in the anti-invasive effect of the omega-3 fatty acid DHA in human melanoma cells. J Dermatol Sci.2016; 84: 149-159.

40. Rossi S, Cordella M, Tabolacci C, et al. TNF-alpha and metalloproteases as key players in melanoma cells aggressiveness. J Exp Clin Cancer Res.2018; 37: 326.

41. Salemi R, Falzone L, Madonna G, et al. MMP-9 as a Candidate Marker of Response to BRAF Inhibitors in Melanoma Patients With BRAF(V600E) Mutation Detected in Circulating-Free DNA. Front Pharmacol.2018; 9: 856

42. Izraely S, Ben-Menachem S, Sagi-Assif $\mathrm{O}$, et al. The metastatic microenvironment: Melanoma-microglia cross-talk promotes the malignant phenotype of melanoma cells. Int J Cancer.2019; 144: 802-817. 